\title{
Computer-Aided Security Proofs for the Working Cryptographer ${ }^{\star}$
}

\author{
Gilles Barthe ${ }^{1}$, Benjamin Grégoire ${ }^{2}$, Sylvain Heraud ${ }^{2}$, \\ and Santiago Zanella Béguelin ${ }^{1}$ \\ ${ }^{1}$ IMDEA Software Institute, Madrid, Spain \\ ${ }^{2}$ INRIA Sophia Antipolis-Méditerranée, France
}

\begin{abstract}
We present EasyCrypt, an automated tool for elaborating security proofs of cryptographic systems from proof sketches - compact, formal representations of the essence of a proof as a sequence of games and hints. Proof sketches are checked automatically using off-the-shelf SMT solvers and automated theorem provers, and then compiled into verifiable proofs in the CertiCrypt framework. The tool supports most common reasoning patterns and is significantly easier to use than its predecessors. We argue that EasyCrypt is a plausible candidate for adoption by working cryptographers and illustrate its application to security proofs of the Cramer-Shoup and Hashed ElGamal cryptosystems.
\end{abstract}

Keywords: Provable security, verifiable security, game-based proofs, Cramer-Shoup cryptosystem, ElGamal encryption.

\section{Introduction}

The game-playing technique [8, 17, 20, is an established methodology for structuring cryptographic proofs. Its essence lies in giving precise mathematical descriptions, referred to as games, of the interaction between adversaries and oracle systems. Proofs are organized as sequences of games, starting from a game that represents a security goal (e.g. indistinguishability against chosen-ciphertext attacks), and proceeding to games that represent security assumptions (e.g. Decision Diffie-Hellman) by successive transformations that can be shown to preserve, or alter only slightly the overall security. In a typical step in a game-based proof the goal is to relate the probability of an event $A$ in a game $G$ to the probability of a possibly different event $A^{\prime}$ in a game $G^{\prime}$. For example, the goal may be to establish an inequality of the form $\operatorname{Pr}[G: A] \leq \operatorname{Pr}\left[G^{\prime}: A^{\prime}\right]+\Delta$, where $\Delta$ is an arithmetic expression that depends on the number of oracle queries made by an adversary. The prevailing practice for proving the validity of such proof steps is to use standard mathematical tools, which interleave reasoning about the semantics of games with information-theoretic or arithmetical arguments.

\footnotetext{
* Partially funded by European Project FP7-256980 NESSoS, French project ANR SESUR-012 SCALP, Spanish project TIN2009-14599 DESAFIOS 10, and Madrid Regional project S2009TIC-1465 PROMETIDOS.
} 
In the code-based approach to the game-playing technique [8,17, games are cast as probabilistic algorithms. The adoption of programming idioms allows to give precise definitions of games, and paves the way for applying programming language methods to justify proof steps rigorously. As anticipated by their proponents, code-based game-playing proofs are amenable to formal verification, and a number of tools provide support for building them. CryptoVerif 11 is a tool for conducting security proofs in a game-based setting in which games are modeled as processes and transitions are justified by means of process-algebraic concepts such as bisimulations. One strength of CryptoVerif, apart from being the first tool to have supported game-based proofs, is that it applies both to protocols and primitives; it has been successfully applied to verify Kerberos 10 . and the Full-Domain Hash (FDH) signature scheme [12. CertiCrypt [6] is another framework that allows for the interactive construction of game-based proofs in the Coq proof assistant [22]. One specificity of CertiCrypt is that proofs can be verified independently and automatically by a small trustworthy checker; it has been successfully applied to verify prominent cryptographic constructions, including OAEP [5], FDH 24, and zero-knowledge protocols [7].

While the developments based on CryptoVerif and CertiCrypt make a convincing case that computer-aided cryptographic proofs are indeed plausible, neither tool has reached a wide audience among cryptographers. In [5], we contrast the high guarantees given by CertiCrypt with the effort and expertise required to build machine-checked proofs, and conclude that cryptographers are unlikely to adopt verifiable security in its current form. In this sense, it can be considered that CryptoVerif and CertiCrypt only provide a partial realization of Halevi's programme of systematically building computer-aided cryptographic proofs [17.

The thesis of this article is that verifiable security can dramatically benefit from automation using state-of-the-art verification technology, and that verifiable game-based proofs can be constructed with only a moderate effort. The thesis is realized with the presentation of EasyCrypt, an automated tool that builds machine-checked proofs from proof sketches, which offer a machine-processable representation of the essence of a security proof. We argue that EasyCrypt is significantly easier to use than previous tools, making an important step towards the adoption of computer-aided security proofs by working cryptographers and hence towards fulfilling Halevi's programme. To substantiate our claim, we present computer-aided proofs of security of Hashed ElGamal encryption and the Cramer-Shoup cryptosystem.

EasyCrypt adopts the principled approach mandated by CertiCrypt to conduct game-based proofs and imposes a clear separation between program verification and information-theoretic reasoning. Transitions between games are justified in two steps: first, one proves logical relations between the games using probabilistic Relational Hoare Logic (pRHL); second, one applies information-theoretic reasoning to derive claims about the probability of events from pRHL judgments. We provide for each step highly effective mechanisms that build upon a combination of off-the-shelf and purpose-specific tools. Specifically, EasyCrypt implements an automated procedure that computes for any pRHL judgment 
a set of sufficient conditions for its validity, known as verification conditions. The outstanding feature of this procedure, and the key to the effectiveness of EasyCrypt, is that verification conditions are expressed in the language of firstorder logic, without any mention of probability, and can be discharged automatically by state-of-the-art tools such as SMT solvers and theorem provers. The verification condition generator is proof-producing, in the sense that it generates Coq files that can be machine-checked using the CertiCrypt framework. Moreover, the connection to CertiCrypt makes it possible to benefit from the expressivity and flexibility of a general-purpose proof assistant for advanced verification goals that fall out of the scope of automated techniques. Additionally, EasyCrypt implements an automated mechanism for proving claims about probability. The mechanism combines some elementary rules to compute (bounds on) probabilities of events - e.g. the probability of a uniformly sampled element to belong to a list - with rules to derive (in)equalities between probabilities of events in games from judgments in pRHL. The combination of these tools with other more mundane features such as a limited form of specification inference for procedures provides substantial leverage towards making verifiable security practical and makes EasyCrypt a plausible candidate for adoption by working cryptographers.

\section{Introductory Example: Hashed ElGamal Encryption}

This section illustrates the application of EasyCrypt to a proof of IND-CPA security of Hashed ElGamal encryption in the Random Oracle Model. The example serves to introduce the notion of proof sketch and to give the reader an idea of the input that the tool expects. It also allows for a preliminary comparison between EasyCrypt and CertiCrypt. We refer the reader to [4 for a proof of the same result in CertiCrypt.

Hashed ElGamal is a variant of ElGamal encryption that does not require plaintexts to be elements of a group. Instead, plaintexts are bitstrings of a certain length $k$ and group elements are mapped into bitstrings using a hash function $H: \mathcal{G} \rightarrow\{0,1\}^{k}$. Let $\mathcal{G}$ be a multiplicative cyclic group of order $q$ with generator $g$. Formally, the scheme is defined by the following triple of algorithms:

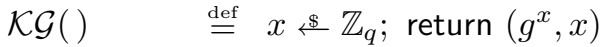

$$
\begin{aligned}
& \mathcal{E}(\alpha, m) \quad \stackrel{\text { def }}{=} y \stackrel{\mathbb{Z}}{=} ; h \leftarrow H\left(\alpha^{y}\right) ; \text { return }\left(g^{y}, h \oplus m\right) \\
& \mathcal{D}(x,(\beta, \zeta)) \stackrel{\text { def }}{=} h \leftarrow H\left(\beta^{x}\right) ; \text { return }(\zeta \oplus h)
\end{aligned}
$$

The security of Hashed ElGamal can be reduced to the Computational DiffieHellman ( $\mathrm{CDH})$ assumption on the underlying group family. This is the assumption that it is hard to compute $g^{x y}$ given $g^{x}$ and $g^{y}$ where $x$ and $y$ are uniformly random elements in $\mathbb{Z}_{q}$. To match the existing proof in CertiCrypt, we exhibit a reduction to the $\mathrm{LCDH}$ assumption, the set version of the $\mathrm{CDH}$ assumption - the reduction from $\mathrm{LCDH}$ to $\mathrm{CDH}$ is immediate. 
Figure 1 shows the sequence of games used to justify the security reduction. This is an essential part of the proof sketch that is input to EasyCrypt, and which is composed of five ingredients 1

1. Type, constant and operator declarations, which introduce the objects manipulated by the scheme. In this case, they include a type for elements of the cyclic group $\mathcal{G}$, constants representing the length of messages $k$, the order of the group $q$ and a generator $g$, and operators denoting the group law and exponentiation, and exclusive or on bitstrings;

2. Axioms, which capture mathematical properties of these objects, and are used by automated tools to check the validity of the proof sketch. We use axioms to state properties of the group law and exponentiation, and the exclusive or operator;

3. Game definitions, where adversaries are specified as abstract procedures with access to oracles. In all games in the figure the hash function $H$ is modeled as a random oracle and the adversary is represented as two procedures $\mathcal{A}_{1}$ and $\mathcal{A}_{2}$ that share state. The procedures representing the adversary are given access to a wrapper $H_{\mathcal{A}}$ for the hash oracle that just stores queries in a list $\boldsymbol{L}_{\mathcal{A}}$ before forwarding them to $H$ :

$$
\begin{aligned}
& H(x) \quad \stackrel{\text { def }}{=} \text { if } x \notin \operatorname{dom}(\boldsymbol{L}) \text { then } h \leftrightarrow\{0,1\}^{k} ; \boldsymbol{L}[x] \leftarrow h \text { end if; return } \boldsymbol{L}[x] \\
& H_{\mathcal{A}}(x) \stackrel{\text { def }}{=} \boldsymbol{L}_{\mathcal{A}} \leftarrow x:: \boldsymbol{L}_{\mathcal{A}} ; m \leftarrow H(x) ; \text { return } m
\end{aligned}
$$

4. Judgments in pRHL. The general form of judgments is $\models \mathrm{G}_{1} \sim \mathrm{G}_{2}: \Psi \Rightarrow \Phi$, where $G_{1}$ and $G_{2}$ are games, and the pre-condition $\Psi$ and the post-condition $\Phi$ are relations on program memories (memories map program variables to values). Pre- and post-conditions are first-order formulae built from relational expressions, in which language expressions are tagged with $\langle 1\rangle$ or $\langle 2\rangle$ to denote their interpretation in the first or second game. We often consider equivalence of memories on a set of variables $X$; we use $=_{X}$ as a shorthand for the formula $\forall x \in X . x\langle 1\rangle=x\langle 2\rangle$;

5. Claims about probability, built from probability quantities (the probability of an event in a game), arithmetic operators, and mathematical relations (e.g. $=,<, \leq$ ). The final statement that expresses the overall security guarantee brought by the proof sketch is usually a claim that upper bounds the probability of adversary success in an initial attack game in terms of the probabilities of one or more adversaries breaking security assumptions.

We briefly comment on the sequence of games in Figure 1, The first and last games encode the IND-CPA and LCDH experiments, respectively. We obtain $\mathrm{G}_{1}$ by inlining the key generation and encryption procedures in the initial game and rearranging instructions so that random choices are made upfront. We prove that games IND-CPA and $G_{1}$ yield identical distributions on the result of the game (denoted by the keyword res). We deduce from this that the probability of the event $b=b^{\prime}$ is the same in both games.

${ }^{1}$ The first two are omitted from the figure. We include an extract of the actual input file for reference in Appendix A 


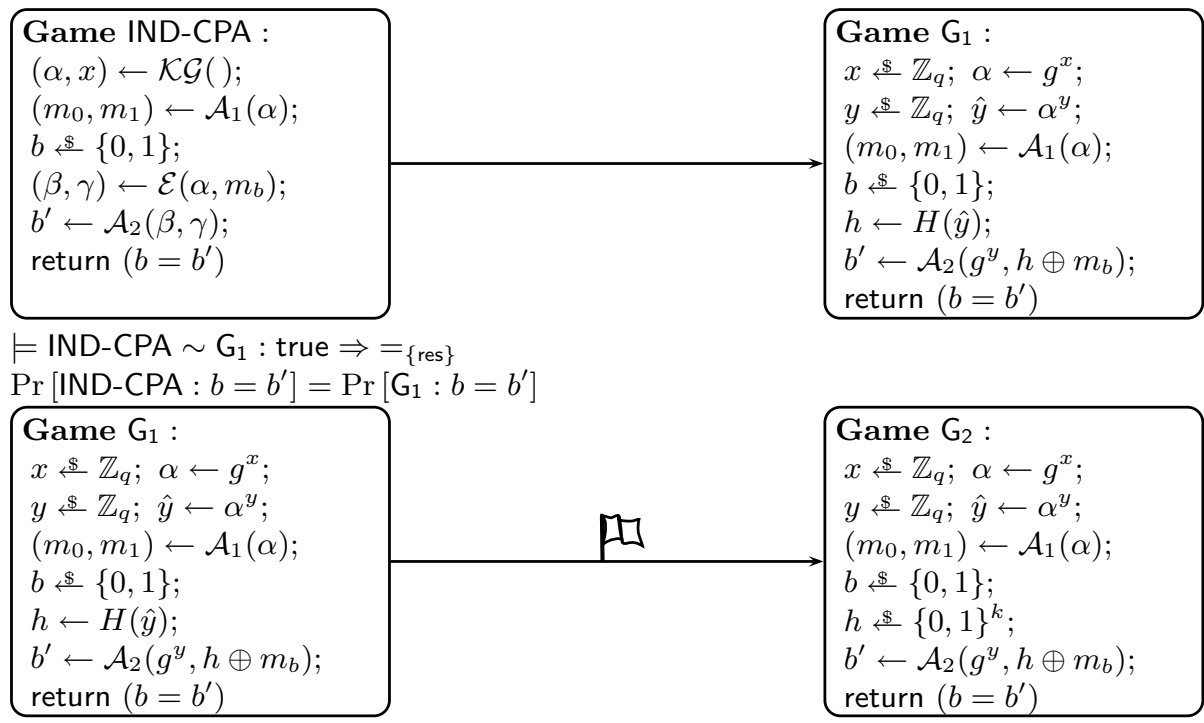

$=\mathrm{G}_{1} \sim \mathrm{G}_{2}:$ true $\Rightarrow\left(\hat{y} \in \boldsymbol{L}_{\mathcal{A}}\right)\langle 1\rangle \leftrightarrow\left(\hat{y} \in \boldsymbol{L}_{\mathcal{A}}\right)\langle 2\rangle \wedge\left(\left(\hat{y} \notin \boldsymbol{L}_{\mathcal{A}}\right)\langle 1\rangle \rightarrow=_{\{\text {res }}\right)$ $\left|\operatorname{Pr}\left[\mathrm{G}_{1}: b=b^{\prime}\right]-\operatorname{Pr}\left[\mathrm{G}_{2}: b=b^{\prime}\right]\right| \leq \operatorname{Pr}\left[\mathrm{G}_{2}: \hat{y} \in \boldsymbol{L}_{\mathcal{A}}\right]$

\begin{tabular}{|c|c|}
\hline 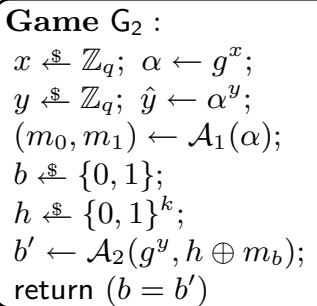 & 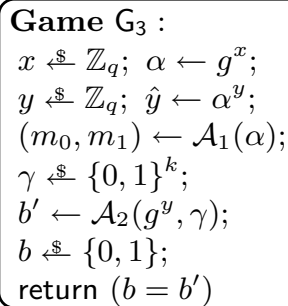 \\
\hline
\end{tabular}

$\models \mathrm{G}_{2} \sim \mathrm{G}_{3}:$ true $\Rightarrow=\left\{\right.$ res $\left., \hat{y}, L_{\mathcal{A}}\right\}$

$\operatorname{Pr}\left[\mathrm{G}_{2}: b=b^{\prime}\right]=\operatorname{Pr}\left[\mathrm{G}_{3}: b=b^{\prime}\right]=1 / 2$

\begin{tabular}{|c|}
\hline 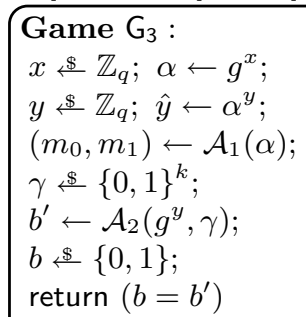 \\
\hline
\end{tabular}

$\operatorname{Pr}\left[\mathrm{G}_{2}: \hat{y} \in \boldsymbol{L}_{\mathcal{A}}\right]=\operatorname{Pr}\left[\mathrm{G}_{3}: \hat{y} \in \boldsymbol{L}_{\mathcal{A}}\right]$

$\models \mathrm{G}_{3} \sim \mathrm{LCDH}:$ true $\Rightarrow\left(\hat{y} \in \boldsymbol{L}_{\mathcal{A}}\right)\langle 1\rangle \leftrightarrow \operatorname{res}\langle 2\rangle$

$\operatorname{Pr}\left[\mathrm{G}_{3}: \hat{y} \in \boldsymbol{L}_{\mathcal{A}}\right]=\operatorname{Pr}\left[\mathrm{LCDH}: g^{x y} \in L\right]$

\begin{tabular}{|c|}
\hline $\begin{array}{l}\text { Game LCDH : } \\
x \leftarrow \mathbb{Z}_{q} ; y \stackrel{\$}{\mathbb{Z}_{q}} ; \\
L \leftarrow B\left(g^{x}, g^{y}\right) \\
\text { return }\left(g^{x y} \in L\right)\end{array}$ \\
\hline $\begin{array}{l}\text { Adversary } \mathcal{B}(\alpha, \beta): \\
\left(m_{0}, m_{1}\right) \leftarrow \mathcal{A}_{1}(\alpha) \\
\gamma \stackrel{\$}{\leftarrow}\{0,1\}^{k} ; \\
b^{\prime} \leftarrow \mathcal{A}_{2}(\beta, \gamma) \\
\text { return } \boldsymbol{L}_{\mathcal{A}}\end{array}$ \\
\hline
\end{tabular}

$$
\mid \operatorname{Pr}\left[\text { IND-CPA }: b=b^{\prime}\right]-\frac{1}{2} \mid \leq \operatorname{Pr}\left[\mathrm{LCDH}: g^{x y} \in L\right]
$$

Fig. 1. Proof sketch of Hashed ElGamal security 
In game $\mathrm{G}_{2}$ we substitute the value $H(\hat{y})$ used to compute the challenge ciphertext by a uniformly chosen value. This only makes a difference if $\mathcal{A}_{1}$ queries $\hat{y}$ to $H$, and this happens with the same probability in either game. Thus, the difference in the probability of any event in these games is bounded by the probability of $\hat{y} \in \boldsymbol{L}_{\mathcal{A}}$ in $\mathrm{G}_{2}$. This can be seen as a semantic variant of the Fundamental Lemma of Game-Playing; the logic allows to dispense with the code instrumentation needed to apply the syntactic counterpart of the lemma.

The transition from $\mathrm{G}_{2}$ to $\mathrm{G}_{3}$ uses a code transformation known as optimistic sampling: instead of sampling $h$ and defining a value $\gamma$ as $h \oplus m_{b}$, we sample $\gamma$ and define $h=\gamma \oplus m_{b}$; we then remove the definition of $h$ as dead code. This transformation is proven admissible within the logic and removes the dependency of the adversary's output from the challenge bit $b$.

The final transition performs the reduction to LCDH by exhibiting an adversary $\mathcal{B}$ that uses $\mathcal{A}$ as a sub-procedure and for which the semantics of games $\mathrm{LCDH}$ and $\mathrm{G}_{3}$ coincide. Finally, from the preceding claims, the advantage of $\mathcal{A}$ can be bounded by the probability of $\mathcal{B}$ in solving LCDH. The resulting proof sketch is about 250 lines long, about 5 times shorter than the proof in CertiCrypt reported in [4] - and arguably much simpler and close to a pen-and-paper proof.

\section{An Overview of EasyCrypt}

Programming Language. Games are modeled as programs in a typed, probabilistic, procedural, imperative language. Types include Booleans, integers, bitstrings, pairs, lists, maps, and user-defined types. Expressions are built from variables and operators in the usual way; for instance, Boolean-valued operators include the usual connectives, equality, list membership, arithmetic comparisons. The commands of the language are defined by the following grammar:

\begin{tabular}{|c|c|}
\hline $\mathcal{I}::=\mathcal{V} \leftarrow \mathcal{E}$ & ascionm \\
\hline $\mathcal{U} \$ \mathcal{D} \mathcal{E}$ & \\
\hline$D \leftarrow D C$ & random sampling \\
\hline if $\mathcal{E}$ then $\mathcal{C}$ else $\mathcal{C}$ & conditional \\
\hline $\mathcal{V} \leftarrow \mathcal{P}(\mathcal{E}, \ldots, \mathcal{E})$ & procedure call \\
\hline $\mathcal{C}::=$ skip & nop \\
\hline $\mathcal{I} ; \mathcal{C}$ & sequence \\
\hline
\end{tabular}

where $\mathcal{V}$ is a set of variables, $\mathcal{P}$ is a set of procedures, and $\mathcal{D E}$ is a set of distribution expressions. For the purpose of this article, distribution expressions are restricted to uniform distributions over specific domains, for instance integers in $\mathbb{Z}_{q}$ or (non-neutral) elements of some group $\mathcal{G}$. Adversaries are modeled as abstract procedures with an interface that specifies the oracles they may query.

Games can be given a semantics as memory distribution transformers, in the style of [6]. Formally, memories are well-typed mappings from variables to values, and the semantics of a game $G$ is a function, denoted $\llbracket G \rrbracket$, that returns for an initial memory $m$ the (sub-)distribution on final memories resulting from executing $\mathrm{G}$ in $m$. Given an initial memory $m$ and an event $A$ (a Boolean expression), we let $\operatorname{Pr}[\mathrm{G}, m: A]$ denote the probability of $A$ w.r.t. the distribution $\llbracket \mathrm{G} \rrbracket m$; we simply write $\operatorname{Pr}[G: A]$ when the initial memory is not relevant. 
Relational Judgments. Pre- and post-conditions in pRHL judgments are firstorder formulae built from relational expressions. Relational expressions are arbitrary Boolean expressions over logical variables and program variables tagged with $\langle 1\rangle,\langle 2\rangle$; the only restriction is that logical variables may only appear quantified. By abuse of notation, we write $e\langle i\rangle$ for the expression $e$ in which all variables have been tagged with $\langle i\rangle$. Let $b$ stand for an arbitrary Boolean expression over tagged and logical variables, then logical formulae are defined by the following grammar:

$$
\Psi, \Phi::=b|\neg \Phi| \Psi \wedge \Phi|\Psi \vee \Phi| \Psi \rightarrow \Phi|\Psi \leftrightarrow \Phi|(\Phi)|\forall x . \Phi| \exists x . \Phi
$$

A logical formula is interpreted as a relation on program memories. For example, the formula $x\langle 1\rangle+y\langle 2\rangle \leq z\langle 1\rangle$ is interpreted as the relation

$$
R=\left\{\left(m_{1}, m_{2}\right) \mid m_{1}(x)+m_{2}(y) \leq m_{1}(z)\right\}
$$

A pRHL judgment $\models \mathrm{G}_{1} \sim \mathrm{G}_{2}: \Psi \Rightarrow \Phi$ is valid iff for any pair of initial memories $m_{1}, m_{2}$ satisfying the pre-condition $\Psi$, the distributions $\llbracket G_{1} \rrbracket m_{1}$ and $\llbracket G_{2} \rrbracket m_{2}$ satisfy the lifting of post-condition $\Phi,\left(\llbracket \mathrm{G}_{1} \rrbracket m_{1}\right) \mathcal{L}(\Phi)\left(\llbracket \mathrm{G}_{2} \rrbracket m_{2}\right)$. The lifting of a relation to a distribution is defined as a max-cut min-flow problem, in the style of [18. Formally, let $\mu_{1}$ be a probability distribution on a set $A$ and $\mu_{2}$ a probability distribution on a set $B$. We define the lifting $\mu_{1} \mathcal{L}(R) \mu_{2}$ of a relation $R \subseteq A \times B$ to $\mu_{1}$ and $\mu_{2}$ as follows 2

$\exists \mu: \mathcal{D}(A \times B) . \pi_{1}(\mu)=\mu_{1} \wedge \pi_{2}(\mu)=\mu_{2} \wedge \forall(a, b): A \times B . \mu(a, b)>0 \Longrightarrow a R b$ where the projections $\pi_{1}(\mu)$ and $\pi_{2}(\mu)$ of $\mu$ are defined as

$$
\pi_{1}(\mu)(a) \stackrel{\text { def }}{=} \sum_{b \in B} \mu(a, b) \quad \pi_{2}(\mu)(b) \stackrel{\text { def }}{=} \sum_{a \in A} \mu(a, b)
$$

Claims about probability can be derived from valid relational judgments by means of the following rules:

$$
\begin{aligned}
& \frac{m_{1} \Psi m_{2} \quad \models \mathrm{G}_{1} \sim \mathrm{G}_{2}: \Psi \Rightarrow \Phi \quad \Phi \rightarrow(A\langle 1\rangle \leftrightarrow B\langle 2\rangle)}{\operatorname{Pr}\left[\mathrm{G}_{1}, m_{1}: A\right]=\operatorname{Pr}\left[\mathrm{G}_{2}, m_{2}: B\right]}[\mathrm{PrEq}] \\
& \frac{m_{1} \Psi m_{2} \quad \models \mathrm{G}_{1} \sim \mathrm{G}_{2}: \Psi \Rightarrow \Phi \quad \Phi \rightarrow(A\langle 1\rangle \rightarrow B\langle 2\rangle)}{\operatorname{Pr}\left[\mathrm{G}_{1}, m_{1}: A\right] \leq \operatorname{Pr}\left[\mathrm{G}_{2}, m_{2}: B\right]}[\text { PrLe }]
\end{aligned}
$$

Automated Proofs of Relational Judgments Most practical verification tools adopt a similar methodology: a weakest precondition (wp) calculus is used to compute from a program and its specification a set of sufficient conditions, known as verification conditions, and these conditions are discharged by automated

${ }^{2}$ For the clarity of presentation, we assume that $A$ and $B$ are discrete and cast our definitions using the usual representation of distributions. However, the tool builds on a monadic representation of distributions, as in [6]. 
tools. Extending the methodology to the logic pRHL is a significant challenge, for two reasons: first, generating verification conditions for a relational program logic is an open topic of research, and second, there is no prior application of the methodology to procedural nor probabilistic programs.

There are at least two natural strategies for defining a wp calculus in a relational setting. The calculus can either operate on both games in lockstep, or else it can operate on each game separately, in the style of self-composition [2]. Both strategies are incomplete: the lockstep wp calculus fails on programs that are not structurally equivalent, whereas self-composition fails to handle random assignments and adversary calls. In order to circumvent these limitations, EasyCrypt implements an alternative approach that mixes both strategies:

1. Calls to non-adversary procedures are eliminated from the games by successive inlining their definitions. In the absence of recursion, the transformation terminates successfully and only adversary calls remain;

2. Random assignments are moved upfront. The resulting code consists of a sequence of random assignments followed by deterministic code, possibly with adversary calls;

3. A relational weakest precondition calculus is applied to the deterministic fragment of the game, using relational specifications to deal with adversary calls. Each adversary specification induces a proof obligation, expressed as a pRHL judgment, on the oracles in its interface. Self-composition is applied to verify the code of oracles with respect to these pRHL judgments. This results in a judgment of the form

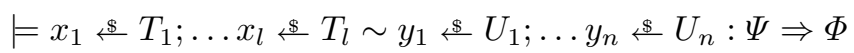

4. A mapping $f: T_{1} \times \cdots \times T_{l} \rightarrow U_{1} \times \cdots \times U_{n}$ is selected, and used to generate the verification condition $\Phi \Rightarrow_{f} \Psi$, defined as

$$
\forall m_{1} m_{2} t_{1} \ldots t_{l} \cdot m_{1} \Psi m_{2} \Longrightarrow m_{1}\{\vec{t} / \vec{x}\} \Phi m_{2}\left\{f\left(t_{1}, \ldots, t_{l}\right) / \vec{y}\right\}
$$

Under specific conditions on $f$, see [23], the validity of $\Phi \Rightarrow_{f} \Psi$ entails the validity of the corresponding pRHL judgment. In practice, it is generally sufficient to require that $f$ is a 1-1 mapping, and taking $f$ as the identity function works most of the time. However, in some cases other mappings must be used. For example, to prove the equivalence between games $\mathrm{G}_{2}$ and $\mathrm{G}_{3}$ in the proof of Hashed ElGamal described in the previous section, it is necessary to prove a judgment like the following:

$$
\models h \leftrightarrow\{0,1\}^{k} ; \gamma \leftarrow h \oplus m_{b} \sim \gamma \$\{0,1\}^{k} ; h \leftarrow \gamma \oplus m_{b}:=_{\left\{m_{b}\right\}} \Rightarrow=_{\{h, \gamma\}}
$$

The wp will stop after computing the weakest precondition for the deterministic fragment of the two programs, yielding

$$
\models h \leftrightarrow\{0,1\}^{k} \sim \gamma \$\{0,1\}^{k}:={ }_{\left\{m_{b}\right\}} \Rightarrow\left(h\langle 1\rangle=\gamma\langle 2\rangle \oplus m_{b}\langle 2\rangle\right)
$$

${ }^{3}$ The memory $m_{1}\{\vec{t} / \vec{x}\}$ maps $x_{i}$ to $t_{i}$ for $i=1 \ldots l$ and $y$ to $m_{1}(z)$ for $z \notin\left\{x_{1} \ldots x_{l}\right\}$. Likewise, $m_{2}\left\{f\left(t_{1}, \ldots, t_{l}\right) / \vec{y}\right\}$ is the memory that maps $y_{i}$ to $\pi_{i}\left(f\left(t_{1}, \ldots, t_{l}\right)\right)$ for $i=1 \ldots n$ and $z$ to $m_{2}(z)$ for $z \notin\left\{y_{1} \ldots y_{n}\right\}$. 
This equivalence is proved in EasyCrypt by providing the bijective function $f(x)=x \oplus m_{b}$ as a witness. The fact that $f$ is bijective is established automatically since $f$ is idempotent. In the general case this is proved by providing also the inverse mapping.

5. Since $\Phi \Rightarrow_{f} \Psi$ is a first-order formula, its validity can be established by off-the-shelf tools. In order to target multiple tools, EasyCrypt generates its verification conditions in the intermediate format of the Why tool [16]. We then use the Simplify prover [15] and the alt-ergo SMT solver [13] to discharge the conditions (although many others provers are supported, including interactive theorem provers such as Coq).

Verification condition generation is incomplete (in the logical sense), and would fail on pRHL judgments where games perform calls to adversaries in a different order. Pleasingly, the strategy is extremely effective in practice - so that we have found no need to implement alternatives for dealing with programs not handled by our approach.

A Mechanized Probabilistic Relational Hoare Logic. EasyCrypt implements a simple tactic language to prove the validity of judgments using rules of the logic and program transformations. The tactics allow the application of twosided rules, which require that the two commands of a judgment have the same shape, and one-sided rules, which operate on only one of the games in a judgment. All language constructs admit both one-sided and two-sided rules, except for random assignments and adversary calls, for which only two-sided rules exist.

The lack of one-sided rules for random assignments and adversary calls limits the applicability of the logic: e.g., it cannot relate the programs $x \leftrightarrow X ; y \leftarrow \mathcal{A}(z)$ and $y \leftarrow \mathcal{A}(z) ; x \leftarrow X$, because instructions are executed in a different order. To mitigate this limitation, EasyCrypt implements program transformations for code motion, allowing to swap instructions that are independent. Moreover, EasyCrypt implements tactics for inlining procedure calls and eagerly/lazily sample random values. Basic tactics can be combined using tacticals to increase automation. The tactic language provides the necessary infrastructure for making most components of EasyCrypt proof-producing, as discussed below.

Reasoning about Failure Events. Game-based proofs often include steps in which it is argued that two games $\mathrm{G}_{1}$ and $\mathrm{G}_{2}$ behave identically unless a designated failure event $F$ occurs. Such transitions are justified using the so-called Fundamental Lemma [20,8], which allows to bound the difference between the probability of an event $A$ in game $\mathrm{G}_{1}$ and a possibly different event $B$ in game $\mathrm{G}_{2}$ by the probability of $F$ in either game. Although a syntactical characterization of this lemma is often used, in which the failure event is represented by a Boolean flag in the code of the games, we state a more general version of the lemma using relational logic. 
Lemma 1 (Fundamental Lemma). Let $\mathrm{G}_{1}, \mathrm{G}_{2}$ be two games and $A, B$, and $F$ be events such that

$$
\models \mathrm{G}_{1} \sim \mathrm{G}_{2}: \Psi \Rightarrow(F\langle 1\rangle \leftrightarrow F\langle 2\rangle) \wedge(\neg F\langle 1\rangle \rightarrow(A\langle 1\rangle \leftrightarrow B\langle 2\rangle))
$$

Then, if $m_{1} \Psi m_{2}$,

1. $\operatorname{Pr}\left[\mathrm{G}_{1}, m_{1}: A \wedge \neg F\right]=\operatorname{Pr}\left[G_{2}, m_{2}: B \wedge \neg F\right]$,

2. $\left|\operatorname{Pr}\left[\mathrm{G}_{1}, m_{1}: A\right]-\operatorname{Pr}\left[\mathrm{G}_{2}, m_{2}: B\right]\right| \leq \operatorname{Pr}\left[\mathrm{G}_{1}, m_{1}: F\right]=\operatorname{Pr}\left[\mathrm{G}_{2}, m_{2}: F\right]$

The hypothesis of the lemma can be checked using the pRHL prover. The key to proving the validity of the judgment is finding an appropriate specification for adversaries. EasyCrypt infers for each adversary call $x \leftarrow \mathcal{A}(\vec{e})$ a relation $\Theta$ and checks the validity of the judgment

$$
\begin{aligned}
\models \mathcal{A} \sim \mathcal{A}: & \left(\neg F\langle 1\rangle \wedge \neg F\langle 2\rangle \wedge={ }_{\operatorname{args}(\mathcal{A})} \wedge \Theta\right) \Rightarrow \\
& (F\langle 1\rangle \leftrightarrow F\langle 2\rangle) \wedge\left(\neg F\langle 1\rangle \rightarrow={ }_{\text {res }\}} \wedge \Theta\right)
\end{aligned}
$$

where $\operatorname{args}(\mathcal{A})$ denotes the set of formal parameters of $\mathcal{A}$. This in turn, requires inferring and checking similar specifications for oracles. Although these heuristically inferred specifications suffice in most cases, the user can choose to prove their own specifications for one or more oracles or adversaries when needed, leaving the tool to infer the rest.

Computing Probabilities. EasyCrypt can prove claims about the probability of events in games using properties of probability (e.g. inclusion-exclusion principle), arithmetic laws, and the rules [PrEq] and [PrLe] above, which allow deriving probability claims from valid relational judgments. We also implement a simple mechanism for computing probability bounds. This mechanism can establish, for instance, that the probability that a value uniformly chosen from a set $T$ is equal to an arbitrary expression is $1 /|T|$, or the probability it belongs to a list of $n$ values is at most $n /|T|$.

Generating Verifiable Evidence. EasyCrypt implements a compiler that turns proof sketches into Coq files that are compatible with the CertiCrypt framework and can be verified using the type checker of Coq. The compiler serves two purposes: first, it significantly increases confidence in proof sketches by producing independently verifiable proofs, and providing means of checking the consistency of the set of axioms used in a proof sketch. Second, it opens the possibility to conduct in a general-purpose proof assistant proof steps that fall out of the scope of automated methods.

We briefly describe the workings of the compiler. The declarations, definitions of games, and axioms of a proof sketch admit an immediate translation into CertiCrypt. The recommended practice is to prove the axioms used by EasyCrypt in CertiCrypt. In most cases, the axioms already exist in CertiCrypt, or are simple consequences of proven facts. Then, using the proof-producing option of the pRHL prover, all judgments of a proof sketch are compiled into pRHL derivations in CertiCrypt. Finally, the compiler generates for each claim in a proof sketch a Coq lemma that may need to be completed manually with justifications of the probability reasoning performed by EasyCrypt. 


\section{Advanced Application: Cramer-Shoup Cryptosystem}

The Cramer-Shoup cryptosystem is a public-key encryption scheme based on ElGamal encryption that gained fame for being the first efficient asymmetric encryption scheme to be proven secure against adaptive chosen-ciphertext attacks under standard assumptions - the length of ciphertexts is just twice the length of ElGamal ciphertexts. Given a cyclic group (family) $\mathcal{G}$ of order $q$ and a keyed hash function $\left\{H_{k}: \mathcal{G}^{3} \rightarrow \mathbb{Z}_{q}\right\}_{k \in K}$ mapping triples of group elements into integers in $\mathbb{Z}_{q}$, key generation, encryption, and decryption are defined as follows:

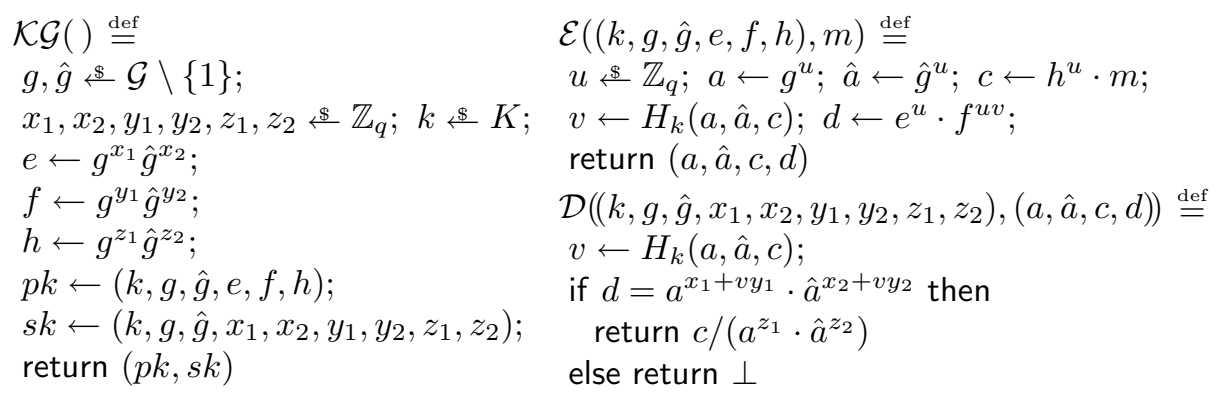

We prove that the Cramer-Shoup cryptosystem is secure against adaptive chosenciphertext attacks (IND-CCA secure) in the standard model assuming the DDH problem is hard in the underlying group family and the hash function $H$ is target collision-resistant (i.e., universal one-way).

Definition 1 (Target Collision-Resistance). Let $\left\{H_{k}: A \rightarrow B\right\}_{k \in K}$ be a keyed family of hash functions. The advantage of an adversary $\mathcal{C}$ against the target collision-resistance of $H$ is defined as

$$
\mathbf{A d v}_{T C R}^{\mathcal{C}} \stackrel{\text { def }}{=} \operatorname{Pr}\left[T C R: H_{k}(x)=H_{k}(y) \wedge x \neq y\right]
$$

where the experiment TCR is defined by means of the following game:

$$
\text { Game TCR : } x \leftarrow \mathcal{C}_{1}() ; k \leftarrow K ; y \leftarrow \mathcal{C}_{2}(k)
$$

Definition 2 (CCA-advantage). Let $(\mathcal{K} \mathcal{G}, \mathcal{E}, \mathcal{D})$ be an asymmetric encryption scheme. The CCA-advantage of an adversary $\mathcal{A}$ limited to $q_{\mathcal{D}}$ decryption queries against the adaptive chosen-ciphertext security of the scheme is defined as

$$
\mathbf{A d v}_{C C A}^{\mathcal{A}}\left(q_{\mathcal{D}}\right) \stackrel{\text { def }}{=}\left|\operatorname{Pr}\left[I N D-C C A: b=b^{\prime}\right]-\frac{1}{2}\right|
$$

\begin{tabular}{|c|c|}
\hline $\begin{array}{l}\text { Game IND-CCA: } \\
(p k, s k) \leftarrow \mathcal{K} \mathcal{G}() ; \\
\left(m_{0}, m_{1}\right) \leftarrow \mathcal{A}_{1}(p k) ; \\
b \stackrel{\$}{\$}\{0,1\} ; \\
\gamma^{*} \leftarrow \mathcal{E}\left(p k, m_{b}\right) ; \gamma_{\text {def }}^{*} \leftarrow \text { true; } \\
b^{\prime} \leftarrow \mathcal{A}_{2}\left(\gamma^{*}\right) ; \\
\text { return }\left(b=b^{\prime}\right)\end{array}$ & $\begin{array}{l}\text { Oracle } \mathcal{D}_{\mathcal{A}}(\gamma): \\
\text { if }\left|\boldsymbol{L}_{\mathcal{D}}\right|<q_{\mathcal{D}} \wedge \neg\left(\gamma_{\text {def }}^{*} \wedge \gamma=\gamma^{*}\right) \text { then } \\
\quad \boldsymbol{L}_{\mathcal{D}} \leftarrow \gamma:: \boldsymbol{L}_{\mathcal{D}} ; \\
\text { return } \mathcal{D}(s \boldsymbol{k}, \gamma) \\
\text { else return } \perp\end{array}$ \\
\hline
\end{tabular}

where the experiment IND-CCA is defined by means of the following game: 
Theorem 1 (Security of Cramer-Shoup). Let $\mathcal{A}$ be an adversary against the IND-CCA security of Cramer-Shoup limited to $q_{\mathcal{D}}$ decryption queries. Then, there exists an algorithm $\mathcal{B}$ for solving the $D D H$ problem in $\mathcal{G}$ and an adversary $\mathcal{C}$ against the target collision-resistance of the hash function $H$ such that

$$
\mathbf{A d v}_{C C A}^{\mathcal{A}}\left(q_{\mathcal{D}}\right) \leq \mathbf{A} \mathbf{d} \mathbf{v}_{D D H}^{\mathcal{B}}+\mathbf{A d v}_{T C R}^{\mathcal{C}}+\frac{q_{\mathcal{D}}^{4}}{q^{4}}+\frac{q_{\mathcal{D}}+2}{q}
$$

Figures 2-4 show a proof sketch of the above theorem in EasyCrypt. The proof follows closely the one presented in [17]; we give only a high-level description here. Game $G_{1}$ in the figure is obtained directly from the IND-CCA game instantiated for Cramer-Shoup by inlining the definitions of the key generation and encryption procedures, propagating assignments, and replacing expressions by equivalent ones. We observe that all verification conditions that ensure the validity of this transformation can be discharged automatically using an SMT solver. This surpasses Halevi's expectations [17], who suggested this transformation be split in three steps so that it could be handled by an automated tool.

We then build a DDH distinguisher $\mathcal{B}$ such that the output distribution on the value of $\left(b=b^{\prime}\right)$ is identical in games $\mathrm{DDH}_{0}$ (where $\mathcal{B}$ receives valid DDH triples) and $G_{1}$, on the one hand, and in games $D H_{1}$ (where $\mathcal{B}$ receives random triples) and $G_{2}$, on the other. In addition, we instrument the decryption oracle in $G_{2}$ to raise a flag bad whenever $\mathcal{A}$ queries for the decryption of a valid ciphertext with $\log _{a} \hat{a} \neq \log _{g} \hat{g}$. We then show using our semantic characterization of the Fundamental Lemma that the difference in the probability of $\left(b=b^{\prime}\right)$ in this game and in game $G_{3}$, where $\mathcal{D}$ rejects such ciphertexts, is bounded by the probability of bad in the latter game. We also change the way $e, f$ and $h$ are computed in a semantics-preserving way. Up to this point, by the triangular inequality we have

$$
\mid \operatorname{Pr}\left[\text { IND-CCA }: b=b^{\prime}\right]-\operatorname{Pr}\left[G_{3}: b=b^{\prime}\right] \mid \leq \mathbf{A d v}_{\mathrm{DDH}}^{\mathcal{B}}+\operatorname{Pr}\left[\mathrm{G}_{3}: \mathbf{b a d}\right]
$$

The next game in the sequence, $\mathrm{G}_{4}$, removes the dependency of the adversary's output from bit $b$ by choosing uniformly $r$ and setting $c=g^{r}$. This requires to be able to compute $z_{2}$ from $\log _{g}(c)=u z+\left(u-u^{\prime}\right) w z_{2}+\log _{g}\left(m_{b}\right)$, which is not possible if $u=u^{\prime}$, but this happens only with probability $1 / q$. We use again the semantic formulation of the Fundamental Lemma to bound the difference in the probability of $\left(b=b^{\prime}\right)$ between $\mathrm{G}_{3}$ and $\mathrm{G}_{4}$ by $1 / q$. After straightforward information-theoretic reasoning we get

$$
\mid \operatorname{Pr}\left[\text { IND-CPA }: b=b^{\prime}\right]-1 / 2 \mid \leq \mathbf{A d v}_{\mathrm{DDH}}^{\mathcal{B}}+2 / q+\operatorname{Pr}\left[\mathrm{G}_{4}: \mathbf{b a d} \wedge u \neq u^{\prime}\right]
$$

We can now move most of the code of the game before the call to $\mathcal{A}_{1}$. This in turn allows to make $d$ random by uniformly choosing $r^{\prime}=\log _{g}(d)$ and defining $x_{2}$ in terms of it, rather than the other way around. Since now the game computes the challenge ciphertext in advance, we can instrument $\mathcal{D}$ to raise a flag $\mathbf{b a d}_{1}$ when the challenge is queried during the first phase of the game. Note that at this point the challenge ciphertext is a 4-tuple of uniformly random elements, 


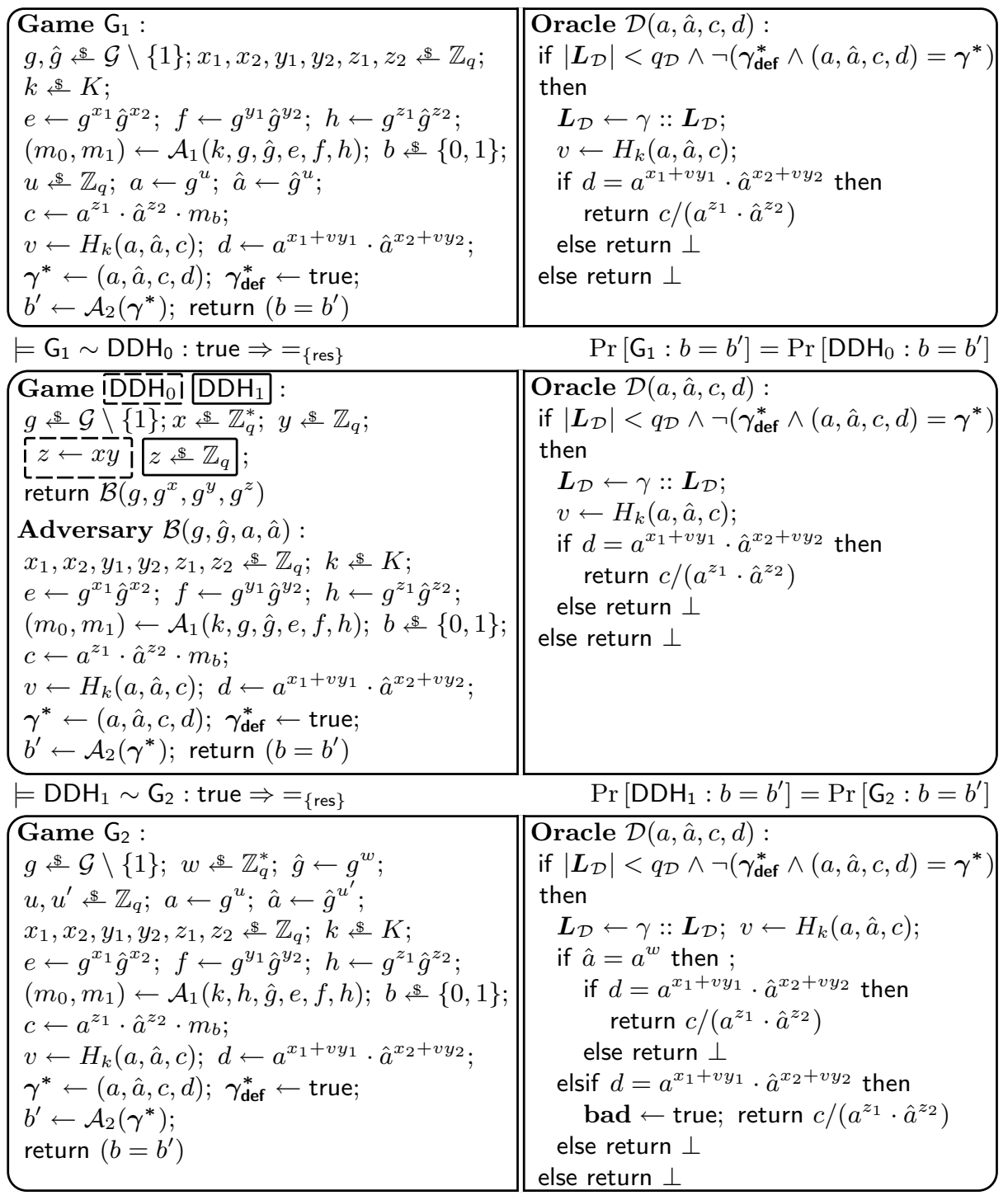

Fig. 2. Proof sketch of the IND-CCA security of the Cramer-Shoup cryptosystem

therefore, the probability of bad $_{1}$ is bounded by $\left(q_{\mathcal{D}} / q\right)^{4}$ - this is achieved by means of an intermediate game, not shown in the figure, that stores the 4 components of queried ciphertexts in different lists, and by independently bounding the probability of each component of the challenge appearing in the corresponding list. Hence, we have

$$
\operatorname{Pr}\left[G_{4}: \operatorname{bad} \wedge u \neq u^{\prime}\right] \leq \operatorname{Pr}\left[G_{5}: \operatorname{bad} \wedge u \neq u^{\prime}\right]+\left(q_{\mathcal{D}} / q\right)^{4}
$$




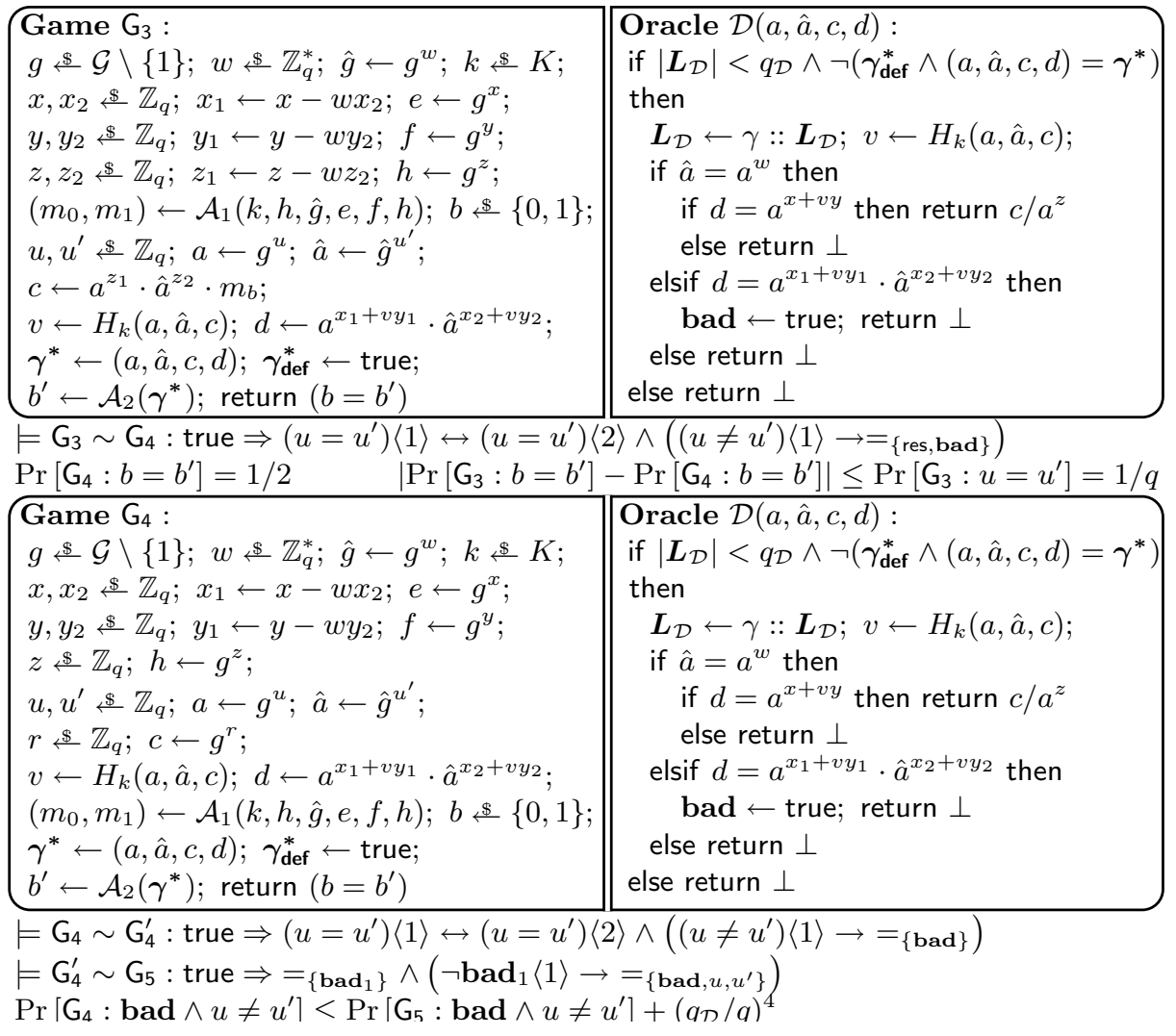

Fig. 3. Proof sketch of the IND-CCA security of the Cramer-Shoup cryptosystem

The decryption oracle in game $\mathrm{G}_{5}$ also raises a flag $\mathbf{b a d}_{2}$ when a valid ciphertext with $H_{k}(a, \hat{a}, c)=H_{k}\left(g^{u}, \hat{g}^{u^{\prime}}, g^{r}\right)$ is queried. Since this leads to a collision, we can build an adversary $\mathcal{C}$ against the TCR of $H$ such that its success probability is lower bounded by the probability of $\mathbf{b a d}_{2}$ being raised in $\mathrm{G}_{5}$. Thus,

$$
\operatorname{Pr}\left[G_{5}: \operatorname{bad} \wedge u \neq u^{\prime}\right] \leq \operatorname{Adv}_{\mathrm{TCR}}^{\mathcal{C}}+\operatorname{Pr}\left[G_{5}: \operatorname{bad} \wedge u \neq u^{\prime} \wedge \neg \operatorname{bad}_{2}\right]
$$

The proof concludes by showing that the probability in $\mathrm{G}_{5}$ of bad being set while bad $_{2}$ is not is bounded by $q_{\mathcal{D}} / q$. This is done by reformulating the test under which $\mathbf{b a d}_{2}$ is set so that it does not depend on $x_{1}, x_{2}, y_{1}, y_{2}$. Therefore, the probability of this test succeeding in any decryption query (under the condition that $u \neq u^{\prime}$ ) is the probability of the adversary guessing a random value in the group, at most $q_{\mathcal{D}} / q$ summing over all queries. The bound in the statement follows. 


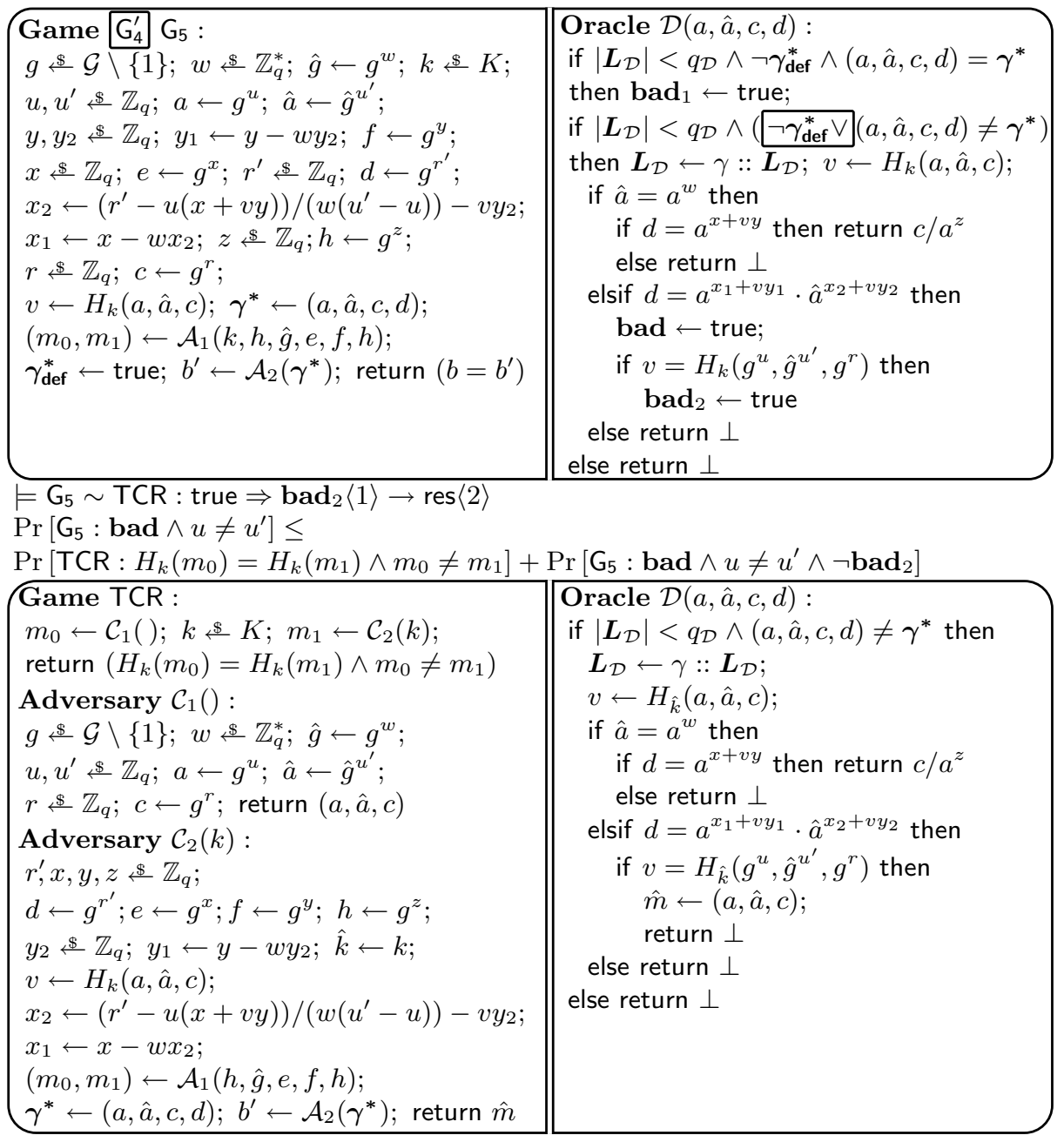

Fig. 4. Proof sketch of the IND-CCA security of the Cramer-Shoup cryptosystem

\section{$5 \quad$ Limitations and Extensions}

EasyCrypt is in its early stages of development; we briefly comment on some of its main limitations and possible extensions:

- Programming language: in comparison with CertiCrypt, the language of EasyCrypt lacks loops, recursive procedures, and drawing from skewed distributions. We do not see the need for extending the current language with recursive procedures. In contrast, we believe that more general forms for sampling and bounded loops are useful and foresee no specific difficulty in 
adding them to the language (note that annotating loops with invariants may be required for verification condition generation);

- Verifiable evidence: EasyCrypt only generates partial verifiable evidence. As there is currently no SMT solver that generates Coq proofs, the verification conditions are admitted in order to make the output derivations checkable by the Coq proof assistant. Making SMT solvers proof-producing is an active subject of research [21], and advances towards this goal shall benefit immediately to EasyCrypt;

- Computation of probability: EasyCrypt generates proof skeletons for claims about probability rather than fully machine-checked proofs. While it is entirely feasible to extend the compiler for justifying more reasonings, a more principled solution would require a tool that can symbolically compute the probability of an event in a distribution.

Further research into the theory of cryptographic proofs, in the line of 3 , is needed to broaden the scope of applications and effectiveness of EasyCrypt. Essential goals include providing a formal account of useful reasoning principles, such as rewinding arguments or coin-fixing, and notions, such as statistical distance, that have not yet been considered in our setting.

There remain ample opportunities to apply methods from programming languages and formal verification to computer-aided cryptographic proofs. We mention two exciting avenues for improving automation in EasyCrypt. The first avenue is to improve our mechanism for inferring relational specifications of adversaries: there is a large body of knowledge on inferring invariants, and it would be beneficial to transpose them to our setting. More speculatively, program synthesis could be used to discover part of the sequence of games needed to conclude a proof, and to build adversaries that justify reductions to cryptographic assumptions. Both specification inference and program synthesis rely on verification condition generation and SMT solving, hence the basic blocks for such an investigation are in place.

Finally, Halevi [17] stresses that "the usefulness of (a) tool will depend crucially on the willingness of the customers (in this case the cryptographic community) to use it", and suggests on this account that an appropriate user interface will be a crucial component of the tool. We fully adhere to his view, and see building such an interface as an important objective for further work.

\subsection{Comparison with CertiCrypt}

Table1 1 compares CertiCrypt and EasyCrypt on various security proofs formalized in both systems. Times are measured on a $2.8 \mathrm{GHz}$ Intel Core 2 Duo processor with $4 \mathrm{~GB}$ of RAM under Mac OS X 10.6.7. For comparison, we show the size and checking time of CertiCrypt proofs extracted from EasyCrypt proof sketches. This is not an altogether fair comparison, because extracted proofs assume as axioms proof obligations checked by automated provers. As an experiment, we completed interactively the extracted proof of security of ElGamal encryption, 
Table 1. Comparison of proof size and checking time between CertiCrypt and EasyCrypt

\begin{tabular}{lrrrrrr}
\hline & \multicolumn{2}{c}{ CertiCrypt } & \multicolumn{2}{c}{ EasyCrypt } & \multicolumn{2}{c}{ Extracted } \\
\hline & Lines & Time & Lines & Time & Lines & Time \\
\hline ElGamal (IND-CPA) & 565 & $45 \mathrm{~s}$ & 190 & $12 \mathrm{~s}$ & 1130 & $23 \mathrm{~s}$ \\
Hashed ElGamal (IND-CPA) & 1255 & $1 \mathrm{~m} 05 \mathrm{~s}$ & 243 & $33 \mathrm{~s}$ & 1772 & $41 \mathrm{~s}$ \\
Full-Domain Hash (EF-CMA) & 2035 & $5 \mathrm{~m} 46 \mathrm{~s}$ & 509 & $1 \mathrm{~m} 26 \mathrm{~s}$ & 2724 & $1 \mathrm{~m} 11 \mathrm{~s}$ \\
Cramer-Shoup (IND-CCA) & $\mathrm{n} / \mathrm{a}$ & $\mathrm{n} / \mathrm{a}$ & 1637 & $5 \mathrm{~m} 12 \mathrm{~s}$ & 5504 & $3 \mathrm{~m} 14 \mathrm{~s}$ \\
OAEP (IND-CPA) & 2451 & $3 \mathrm{~m} 27 \mathrm{~s}$ & $\mathrm{n} / \mathrm{a}$ & $\mathrm{n} / \mathrm{a}$ & $\mathrm{n} / \mathrm{a}$ & $\mathrm{n} / \mathrm{a}$ \\
OAEP (IND-CCA) & 11162 & $37 \mathrm{~m} 32 \mathrm{~s}$ & $\mathrm{n} / \mathrm{a}$ & $\mathrm{n} / \mathrm{a}$ & $\mathrm{n} / \mathrm{a}$ & $\mathrm{n} / \mathrm{a}$ \\
\hline
\end{tabular}

thus obtaining a full proof verifiable under Coq. The resulting proof is 1173 long (meaning that only 43 lines are needed to prove in Coq the proof obligations checked by automated provers) and takes 25 s to check.

\section{Conclusion}

Computer-aided verification of cryptographic protocols in the symbolic model is an established field of research: robust tools are available and have been used successfully to analyze realistic protocols (e.g. [1,9, 14, 19, ). In contrast, there is little prior work on computer-aided cryptographic proofs in the computational model. The importance of such proofs was suggested independently by Bellare and Rogaway [8] and, more explicitly, by Halevi [17], who convincingly argues that they can be viewed as the "natural next step along the way of viewing cryptographic proofs as a sequence of probabilistic games". To date, there are two main tools for computer-aided cryptographic proofs: CertiCrypt, which favors generality and verifiable proofs, and CryptoVerif, which favors automation. We have presented EasyCrypt, a new tool which provides the first flexible and automated framework for building machine-checkable cryptographic proofs, and illustrated its use through computer-aided security proofs of Hashed ElGamal encryption in the Random Oracle Model and the Cramer-Shoup cryptosystem in the standard model. These examples demonstrate that proofs in EasyCrypt are significantly easier and faster to build than in any previous tool, while providing guarantees similar to CertiCrypt. Overall, we believe that EasyCrypt makes an important step towards the adoption of computer-aided proofs by working cryptographers.

Acknowledgments. We are grateful to Daniel Hedin and Anne Pacalet for their participation in the initial phases of the project, to Yassine Lakhnech and David Pointcheval for useful discussions, and to the anonymous reviewers for their insightful comments.

\section{References}

1. Backes, M., Maffei, M., Unruh, D.: Computationally sound verification of source code. In: 17th ACM Conference on Computer and Communications Security, CCS 2010, pp. 387-398. ACM, New York (2010) 
2. Barthe, G., D'Argenio, P., Rezk, T.: Secure information flow by self-composition. In: 17th IEEE Workshop on Computer Security Foundations, CSFW 2004, pp. 100-114. IEEE Computer Society, Washington (2004)

3. Barthe, G., Daubignard, M., Kapron, B., Lakhnech, Y.: Computational indistinguishability logic. In: 17th ACM Conference on Computer and Communications Security, CCS 2010, pp. 375-386. ACM, New York (2010)

4. Barthe, G., Grégoire, B., Heraud, S., Zanella Béguelin, S.: Formal certification of ElGamal encryption. A gentle introduction to CertiCrypt. In: Degano, P., Guttman, J., Martinelli, F. (eds.) FAST 2008. LNCS, vol. 5491, pp. 1-19. Springer, Heidelberg (2009)

5. Barthe, G., Grégoire, B., Lakhnech, Y., Zanella Béguelin, S.: Beyond provable security verifiable IND-CCA security of OAEP. In: Kiayias, A. (ed.) CT-RSA 2011. LNCS, vol. 6558, pp. 180-196. Springer, Heidelberg (2011)

6. Barthe, G., Grégoire, B., Zanella Béguelin, S.: Formal certification of code-based cryptographic proofs. In: 36th ACM SIGPLAN-SIGACT Symposium on Principles of Programming Languages, POPL 2009, pp. 90-101. ACM, New York (2009)

7. Barthe, G., Hedin, D., Zanella Béguelin, S., Grégoire, B., Heraud, S.: A machinechecked formalization of Sigma-protocols. In: 23rd IEEE Computer Security Foundations Symposium, CSF 2010, pp. 246-260. IEEE Computer Society, Los Alamitos (2010)

8. Bellare, M., Rogaway, P.: The security of triple encryption and a framework for code-based game-playing proofs. In: Vaudenay, S. (ed.) EUROCRYPT 2006. LNCS, vol. 4004, pp. 409-426. Springer, Heidelberg (2006)

9. Bhargavan, K., Fournet, C., Gordon, A.D.: Modular verification of security protocol code by typing. In: 37th ACM SIGPLAN-SIGACT Symposium on Principles of Programming Languages, POPL 2010, pp. 445-456. ACM, New York (2010)

10. Blanchet, B., Jaggard, A.D., Scedrov, A., Tsay, J.K.: Computationally sound mechanized proofs for basic and public-key Kerberos. In: 15th ACM Conference on Computer and Communications Security, CCS 2008, pp. 87-99. ACM, New York (2008)

11. Blanchet, B.: A computationally sound mechanized prover for security protocols. In: 27th IEEE Symposium on Security and Privacy, S\&P 2006, pp. 140-154. IEEE Computer Society, Los Alamitos (2006)

12. Blanchet, B., Pointcheval, D.: Automated security proofs with sequences of games. In: Dwork, C. (ed.) CRYPTO 2006. LNCS, vol. 4117, pp. 537-554. Springer, Heidelberg (2006)

13. Conchon, S., Contejean, E., Kanig, J., Lescuyer, S.: CC(X): Semantic combination of congruence closure with solvable theories. Electronic Notes in Theoretical Computer Science 198(2), 51-69 (2008)

14. Cremers, C.: The scyther tool: Verification, falsification, and analysis of security protocols. In: Gupta, A., Malik, S. (eds.) CAV 2008. LNCS, vol. 5123, pp. 414-418. Springer, Heidelberg (2008)

15. Detlefs, D., Nelson, G., Saxe, J.B.: Simplify: A theorem prover for program checking. Tech. Rep. HPL-2003-148, HP Laboratories Palo Alto (2003)

16. Filliâtre, J.C.: The WHY verification tool: Tutorial and Reference Manual Version 2.28 (2010), http://why.lri.fr

17. Halevi, S.: A plausible approach to computer-aided cryptographic proofs. Cryptology ePrint Archive, Report 2005/181 (2005)

18. Jonsson, B., Yi, W., Larsen, K.G.: Probabilistic extensions of process algebras. In: Bergstra, J., Ponse, A., Smolka, S. (eds.) Handbook of Process Algebra, pp. 685-710. Elsevier, Amsterdam (2001) 
19. Paulson, L.C.: The inductive approach to verifying cryptographic protocols. J. of Comput. Secur. 6(1-2), 85-128 (1998)

20. Shoup, V.: Sequences of games: a tool for taming complexity in security proofs. Cryptology ePrint Archive, Report 2004/332 (2004)

21. Stump, A.: Proof checking technology for satisfiability modulo theories. Electr. Notes Theor. Comput. Sci. 228, 121-133 (2009)

22. The Coq development team: The Coq Proof Assistant Reference Manual Version 8.3 (2010), http://coq.inria.fr

23. Zanella Béguelin, S.: Formal Certification of Game-Based Cryptographic Proofs. Ph.D. thesis, Ecole Nationale Supérieure des Mines de Paris - Mines ParisTech (2010)

24. Zanella Béguelin, S., Grégoire, B., Barthe, G., Olmedo, F.: Formally certifying the security of digital signature schemes. In: 30th IEEE Symposium on Security and Privacy, S\&P 2009, pp. 237-250. IEEE Computer Society, Los Alamitos (2009)

\section{A Input File for the Proof of Security of Hashed ElGamal}

The following is an extract taken from the EasyCrypt input file corresponding to the proof of IND-CPA security of Hashed ElGamal described in Section 2.

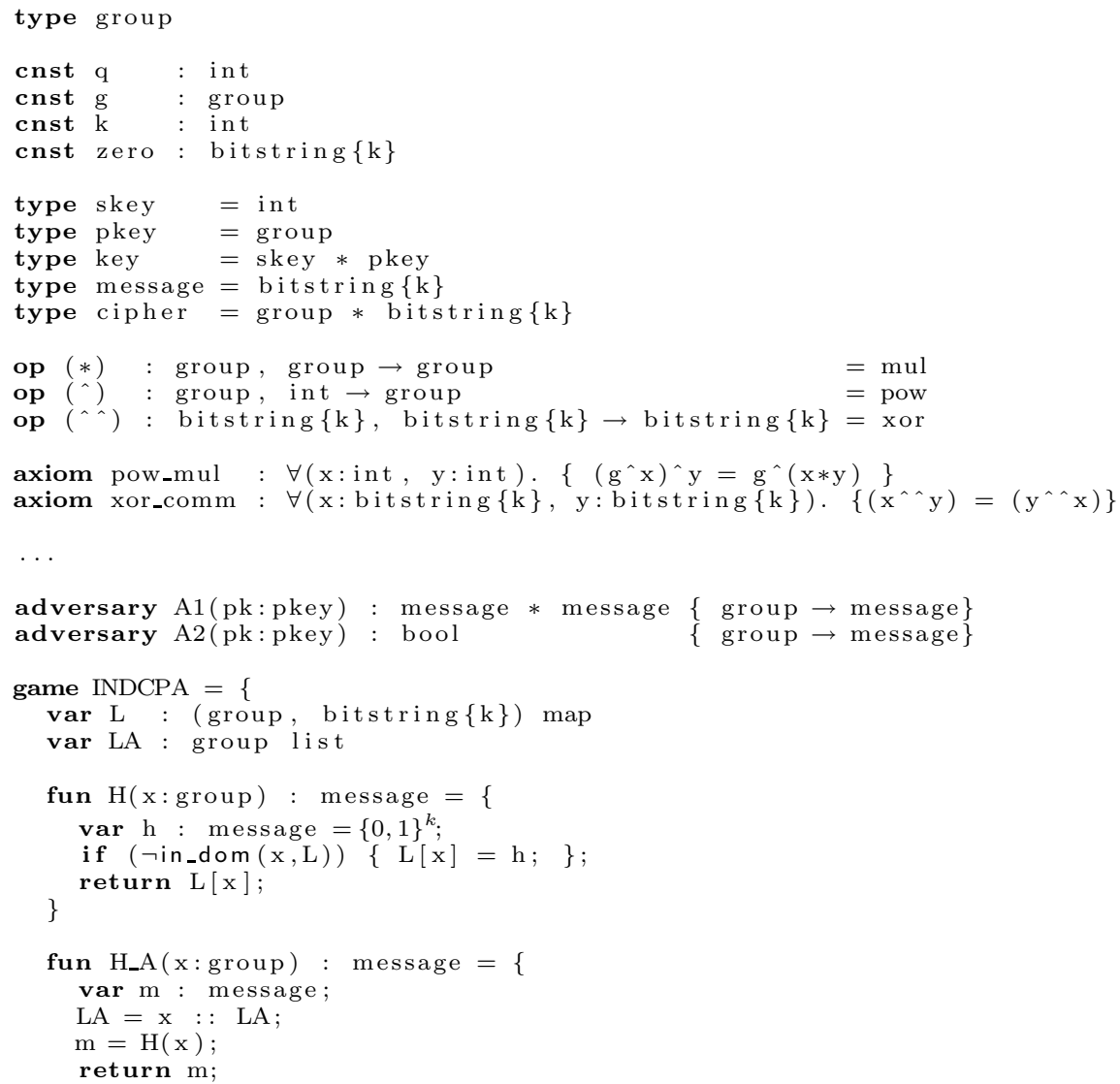




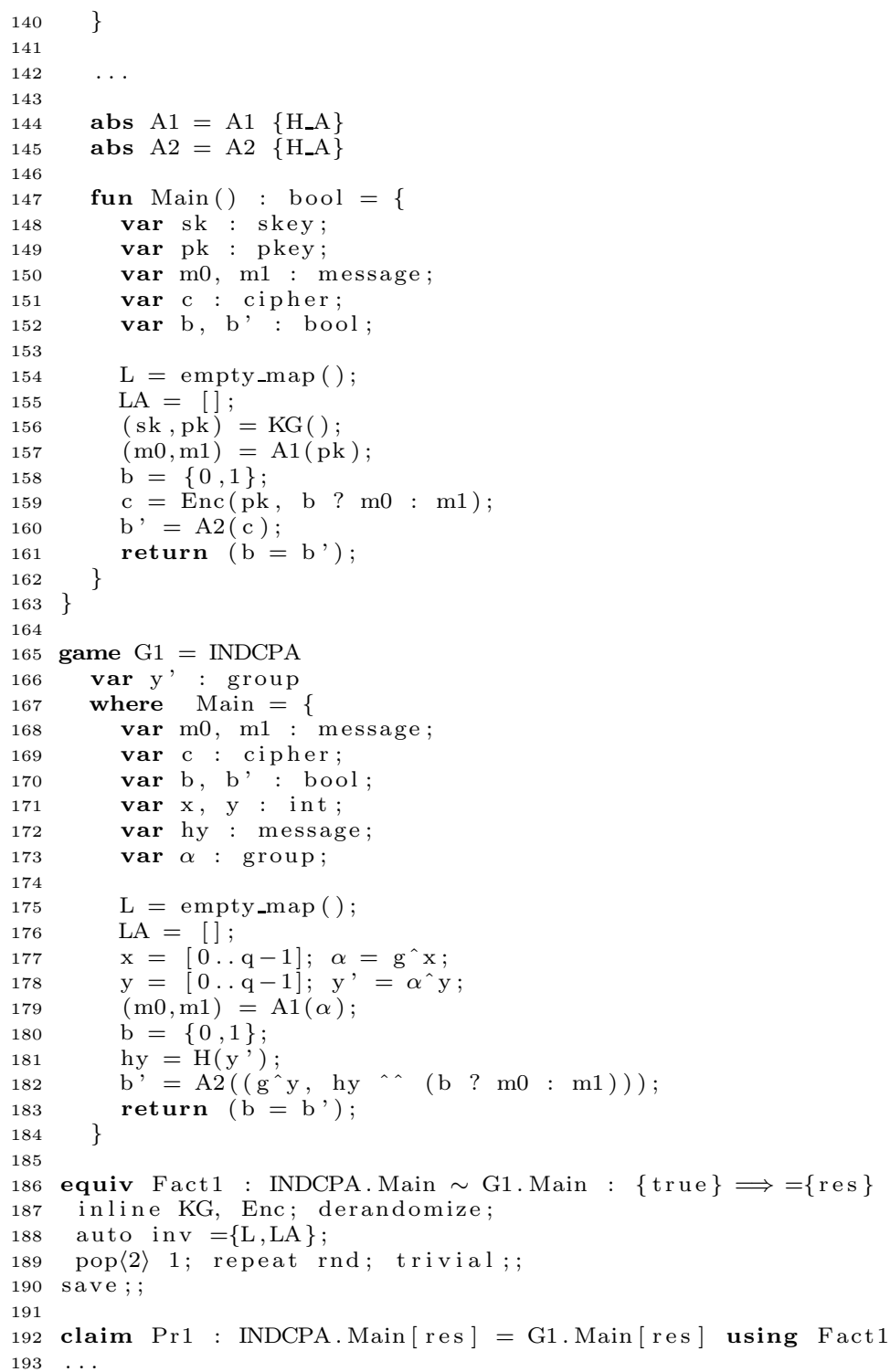

